\section{Guagueira induzida por aripiprazol e tratada com risperidona}

\author{
Aripiprazole-induced stuttering treated \\ with risperidone
}

de base. A dose de 30mg foi reintroduzida e foi associado $1 \mathrm{mg} /$ dia de risperidona. Houve melhora parcial da gagueira e, após aumento para $2 \mathrm{mg} / \mathrm{dia}$, observamos remissão do quadro clínico após dois meses.

Durante esse período, a paciente não apresentou nenhum sinal e/ou sintoma de infecção, doença neurológica ou história de traumatismo crânio-encefálico. Exames laboratoriais de rotina (hemograma, TSH, uréia, creatinina, EAS) não apresentaram alterações dignas de nota.

Discussão: o aripiprazol é um neuroléptico com farmacodinâmica muito peculiar, apresentando-se como agonista parcial dos receptores dopaminérgicos do tipo 2 (D2). Baseando-se na teoria de disfunção dopaminérgica para a gagueira, a ação peculiar do neuroléptico, estimulando os receptores em determinadas regiões, pode explicar o sintoma observado ${ }^{1,4}$.

A risperidona, assim como outros neurolépticos (como, por exemplo, a pimozida e a olanzapina) foram avaliados como fármacos para tratar a gagueira, bloqueando os receptores D2 ${ }^{1}$. A risperidona já foi testada em estudos caso-controle onde a administração de baixas doses $(0,5$ a 2,0mg) demonstraram redução da gravidade da gagueira em 16 indivíduos ${ }^{3}$. Em nosso caso, receptores D2 de vias de neurotransmissão ainda não elucidadas estariam sendo estimulados pelo aripiprazol, e o bloqueio desses receptores com a introdução da risperidona teriam revertido o quadro de gagueira.

Apesar de se tratar de um caso clínico, sua importância se deve a um melhor esclarecimento na fisiopatologia da gagueira, dos efeitos colaterais do aripiprazol e da reafirmação da risperidona como uma opção de tratamento. Porém, mais estudos tornam-se necessários para uma melhor elucidação das diversas variáveis e dúvidas que acompanham o assunto.

Felipe Filardi da Rocha Hospital Galba Velloso, Belo Horizonte (MG), Brasil Faculdade de Medicina do Vale do Aço, Ipatinga (MG), Brasil Departamento de Farmacologia Bioquímica e Molecular, Instituto de Ciências Biológicas, Universidade Federal de Minas Gerais (UFMG), Belo Horizonte (MG), Brasil

aumento da dose do neuroléptico, uma tentativa de redução da dose foi tentada com melhora da gagueira, mas piora do transtorno

Financiamento e conflito de interesses

\begin{tabular}{|c|c|c|c|c|c|c|c|}
\hline $\begin{array}{l}\text { Membro do } \\
\text { grupo de } \\
\text { autores }\end{array}$ & Local de trabalho & $\begin{array}{l}\text { Verba de } \\
\text { pesquisa }^{1}\end{array}$ & $\begin{array}{c}\text { Outro apoio à } \\
\text { pesquisa ou } \\
\text { educação médica } \\
\text { continuada }\end{array}$ & $\begin{array}{c}\text { Honorários de } \\
\text { palestrante }\end{array}$ & $\begin{array}{l}\text { Participação } \\
\text { acionária }\end{array}$ & $\begin{array}{l}\text { Consultor/ } \\
\text { Conselho } \\
\text { consultivo }\end{array}$ & Outro $^{3}$ \\
\hline Felipe Filardi da & Hospital Galba Velloso & - & - & - & - & - & - \\
\hline \multirow[t]{2}{*}{ Rocha } & $\begin{array}{c}\text { Faculdade de Medicina } \\
\text { do Vale do Aço }\end{array}$ & & & & & & \\
\hline & UFMG & & & & & & \\
\hline
\end{tabular}

* Modesto

** Significativa

*** Significativa. Montantes fornecidos à instituição do autor ou a colega para pesquisa onde o autor tem participação, não diretamente ao autor

Nota: UFMG = Universidade federal de Minas Gerais.

Para mais informações, ver Instruções aos autores.

\section{Referências}

1. Vila-Nova C, Queirós F, Fortaleza T, Lucena R. Pharmacologic treatment of stuttering: evidences and controversies. J Bras Psiquiatr. 2006;55(3):244-8.

2. de Andrade CR, Sassi FC, Juste F, de Mendonça LI. Persistent developmental stuttering as a cortical-subcortical dysfunction: evidence from muscle activation. Arq Neuropsiquiatr. 2008;66(3B):659-64.
3. Maguire GA, Riley GD, Franklin DL,Gottchalk LA. Risperidone for the treatment of stuttering. J Clin Psychopharmacol. 2000;20(4):479-82.

4. da Rocha FF, Correa H. Successful augmentation with aripiprazole in clomipramine-refractory obsessive-compulsive disorder. Prog Neuropsychopharmacol Biol Psychiatry. 2007;31(7):1550-1. 